\title{
Variable-Rate Quadtree-segmented Block Truncation Coding for Color Image Compression
}

\author{
Wu-Lin Chen ${ }^{1}$, Yu-Chen $\mathrm{Hu}^{1}$, Kuo-Yu Liu ${ }^{2}$, Chun-Chi Lo ${ }^{3}$ and Chia-Hsien Wen ${ }^{1}$ \\ ${ }^{1}$ Department of Computer Science and Information Management, \\ Providence University, 200 Chung Chi Rd., Taichung 43301, Taiwan \\ ${ }^{2}$ Department of Computer Science and Communication Engineering, \\ Providence University, 200 Chung Chi Rd., Taichung 43301, Taiwan \\ ${ }^{3}$ Department of Computer Science and Information Engineering, \\ Providence University, 200 Chung Chi Rd., Taichung 43301, Taiwan \\ \{wlchen, ychu,cclo, kyliu, chwen\}@pu.edu.tw
}

\begin{abstract}
A novel color image compression scheme based on block truncation coding is proposed in this paper. In this scheme, the quadtree segmentation technique is employed to partition the color image into variable-sized image blocks according to its block activities. Then, the block truncation coding scheme with the bit map omission technique is employed to encode the image blocks. According to the experimental results, the proposed scheme significantly cuts down the bit rates while keeping good reconstructed image qualities of the compressed color images.
\end{abstract}

Keywords: color image compression, block truncation coding, quadtree segmentation, bit map omission

\section{Introduction}

In the past few years, digital images are widely used in most multimedia applications. Generally, the digital images are stored in the compressed forms instead of the raw format to save the transmission bandwidth and the storage cost. Till now, several image coding schemes, such as vector quantization [1,8], color image quantization [9, 15], block truncation coding (BTC) [16, 18], sub-band coding, JPEG, and JPEG 200, had been proposed. Among them, BTC is a commonly used image coding scheme for image compression.

BTC was first proposed to compress the grayscale images by Delp and Mitchell in 1979 [16]. It is also known as the moment preserving block truncation coding (MPBTC) because it preserves the first and second moments of image blocks. To encode each grayscale image, each image is partitioned into a set of non-overlapped image blocks of $n \times n$ pixels. The quantization threshold of each image block is calculated. According to the quantization threshold, all the pixels in each image block are classified into two groups. The grouping information of each image block is stored in the binary bit map of $n \times n$ bits. The quantization levels of these two groups are then computed. The compressed codes of each image block consist of one bit map and two quantization levels.

Till now, several modified BTC schemes, such as the third moment preserving quantization scheme [16], the mean squared error optimal quantization scheme [18], the absolute moment block truncation coding scheme (AMBTC) [19], and the generalized moment preserving quantization scheme [20], had been introduced. These schemes were introduced to hold different block properties. Among them, AMBTC had been proven to be the optimal scheme as far as the squared Euclidean distance measurement is concerned. 
In 1984, Lema and Mitchell proposed the absolute moment block truncation coding scheme [19] for both grayscale and color image coding. In this scheme, the color image to be compressed is decomposed into three grayscale images. Then, each grayscale image is sequentially compressed. In other words, the compressed codes of a color image block by using AMBTC consist of three bit maps and six quantization levels.

Basically, BTC has the advantage of providing good image qualities of the compressed images. In addition, the image encoding/decoding procedures of BTC are very simple. That is why BTC is particularly suitable for real time and low computational cost multimedia applications. However, BTC consumes a high number of bit rates for image coding.

To reduce the bit rates of BTC for grayscale image compression, some schemes [21, 22] that employed the representative patterns to encode bit maps had been proposed. In addition, the interpolation of the bit map $[23,24]$ had been introduced. In these schemes, only partial bit maps are transmitted to the receiver. Then, the receiver executes the interpolation process to recover the bit maps that were not transmitted. Some BTC-based image coding schemes which used the quadtree segmentation technique [25, 26] had been proposed to cut down the required bit rates. Besides, the predictive coding technique had been employed to design BTC-based image coding schemes [27, 28]. Both the quadtree segmentation technique and the predictive coding technique exploit the spatial similarity among neighboring pixels in the images.

To cut down the bit rates of BTC for color image compression, the single bit map BTC scheme had been introduced in 1992 [29]. In single bit map BTC, one common bit map instead of three bit maps is taken to represent each color image block. Three rules, the majority rule, the luminance rule, and the weighted rule, had been designed to generate the common bit map for each color image block. The compressed codes of each color image block consist of one bit map and six quantization levels. The authors suggested that the weighted rule should be used to generate the common bit map because it achieves the best reconstructed image qualities according to the experimental results.

Currently, some improved image coding schemes [30, 31] based on single bit map BTC had been proposed. The color image compression by using moment preserving and block truncation coding technique (CICMPBTC) [30] had been proposed. The high correlations among quantization levels are exploited and the spectral identification code is designed to record the total number of quantizaiton levels transmitted to the receiver. In addition, the two-layer AMBTC scheme (TLAMBTC) [31] for color image compression had been proposed. TLAMBTC employs the single bit map AMBTC to compress the color images in the first layer. Then, the quantization levels are coded by AMBTC in the second layer. If the quantization levels to be coded in the second layer are very close to one another, the mean of the quantization levels is used to encode them.

In 2008, Hu et al., proposed a novel color image coding scheme based on block prediction coding and AMBTC (AMBTC-BPC) [32]. The goal of AMBTC-BPC is to reduce the bit rates of AMBTC for color image coding. The block prediction coding technique is employed to exploit the block similarity. In addition, the bit map omission technique and the coding of the quantization levels are employed in AMBTC-BPC.

Several color image coding schemes based on BTC had been proposed so far. To further cut down the required bit rates while keeping good image qualities, a novel image coding scheme based on AMBTC is proposed in this paper. The rest of the paper is organized as follows. In Section 2, the review of the AMBTC scheme will be given. In Section 3, the newly proposed scheme will be introduced. The experimental results will be given in Section 4 to show if the proposed scheme indeed produces better performance. Finally, some conclusions will be given in Section 5 . 


\section{Review on Absolute Moment Block Truncation Coding}

In 1984, Lema and Mitchell proposed the absolute moment block truncation coding scheme [19] for grayscale and color image compression. The goal of AMBTC is to preserve the mean and the first absolute central moment of image blocks. Each grayscale image to be compressed is divided into a set of non-overlapping image blocks of $n \times n$ pixels. Each $n \times n$ image block can be viewed as an image vector of $k$ dimensions, where $k$ $=n \times n$. Each image block is sequentially processed in the order of left-to-right and top-to-down.

To compress each image block $x$, the block mean $(\bar{x})$ is calculated according to the following equation:

$$
\bar{x}=\frac{1}{k} \sum_{i=1}^{k} x_{i} .
$$

To generate the bit map ( $B M)$ of $x$, all the pixels in $x$ are classified into two groups according to $\bar{x}$. If the intensity of one pixel is less than $\bar{x}$, it is classified as the first group and a corresponding bit with value 0 is stored in $B M$. Otherwise, it is classified as the second group and a corresponding bit with value 1 is stored in $B M$.

Pixels in the same group will be encoded by the same quantization level. Let $a$ and $b$ denote the quantization levels in the first and the second groups, respectively. These two quantization levels can be computed according to the following equations:

$$
\begin{aligned}
& a=\frac{1}{k-q} \times \sum_{x_{i}<x} x_{i}, \text { and } \\
& b=\frac{1}{q} \times \sum_{x_{i} \geq x} x_{i} .
\end{aligned}
$$

Here, $q$ stands for the number of pixels whose values are greater than or equal to $\bar{x}$.

Each compressed image block forms a trio $(a, b, B M)$ where each quantization level is stored in 8 bits. For each compressed block in AMBTC, a total of $(8+8+k)$ bits are needed to store the compressed codes $(a, b, B M)$. The required number of bit rates of AMBTC equals $(8+8+k) / k$ bpp. For example, the number of bit rates of the AMBTC scheme equals 2 bpp when the block size $k$ is set to 16 .

In the image decoding procedure, each image block is to be rebuilt by using the received trio $(a, b, B M)$. One pixel is reconstructed by quantization level $a$ if a corresponding bit valued 0 is found in the bit map $B M$. Otherwise, it is recovered by quantization level $b$. After each pixel is recovered by either the quantization level $a$ or $b$ according to its retrieved bit map, the image block is then reconstructed. When each image block is sequentially recovered by using the above-mentioned step, the whole compressed image of the AMBTC scheme can be generated.

\begin{tabular}{|c|c|c|c|}
\hline 142 & 88 & 70 & 52 \\
\hline 152 & 118 & 92 & 78 \\
\hline 168 & 158 & 120 & 99 \\
\hline 188 & 172 & 145 & 114 \\
\hline
\end{tabular}

\section{Figure 1. Image Block of $4 \times 4$ Pixels}

An example of the image encoding procedure is described in the following. Figure 1 shows the original image block of $4 \times 4$ pixels. The block mean is first computed by using Eq. (1). In this example, the block mean equals 122.25. According to the block mean, the 
bit map as shown in Figure 2(a) can then be generated. The quantization levels for these two groups are calculated by using Eqs. (2) and (3), respectively. These two quantization levels are 92 and 161, respectively. Finally, the compressed trio (92, 161, $\left.(1000100011001110)_{2}\right)$ is sent to the receiver.

To decode the compressed image, the compressed trio $\left(92,161,(1000100011001110)_{2}\right)$ is extracted. To recover each pixel, each bit in of the bit map is sequentially checked to recover the compressed pixel and the reconstructed image block is shown in Figure 2(b).

\begin{tabular}{|l|l|l|l|}
\hline 1 & 0 & 0 & 0 \\
\hline 1 & 0 & 0 & 0 \\
\hline 1 & 1 & 0 & 0 \\
\hline 1 & 1 & 1 & 0 \\
\hline
\end{tabular}

(a) Bit map

\begin{tabular}{|c|c|c|c|}
\hline 161 & 92 & 92 & 92 \\
\hline 161 & 92 & 92 & 92 \\
\hline 161 & 161 & 92 & 92 \\
\hline 161 & 161 & 161 & 92 \\
\hline
\end{tabular}

(b) Decoded image block

Figure 2. The Image Encoding/Decoding Example

\section{The Proposed Scheme}

In this section, a quadtree-segmented AMBTC scheme with bit map omission is introduced. We call it AMBTC-QTBO. The quadtree segmentation technique will be introduced in Section 3.1. Next, the bit map omission technique will be discussed in Section 3.2. Finally, the detailed description of AMBTC-QTBO will be given in Section 3.3.

\subsection{The Quadtree Segmentation Technique}

The quadtree segmentation technique is a hierarchical segmentation technique that partitions one given image into variable-sized blocks based on the quadtree structure. Typically, the top-down approach is used because of its simplicity and flexibility. A series of binary decisions up to a set of different threshold values are made when the quadtree segmentation process is executed.

Usually, a block type classifier is employed to determine the block type. The block type classifier employs some statistical information such as block variance, averaged block absolute error, and so on, to determine the block activity. By choosing adequately controlling thresholds, different statistical measurements can be employed to control the quadtree segmentation process.

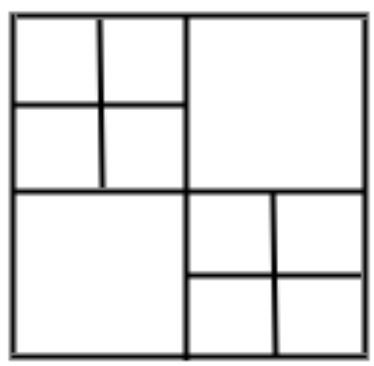

(a) Original image

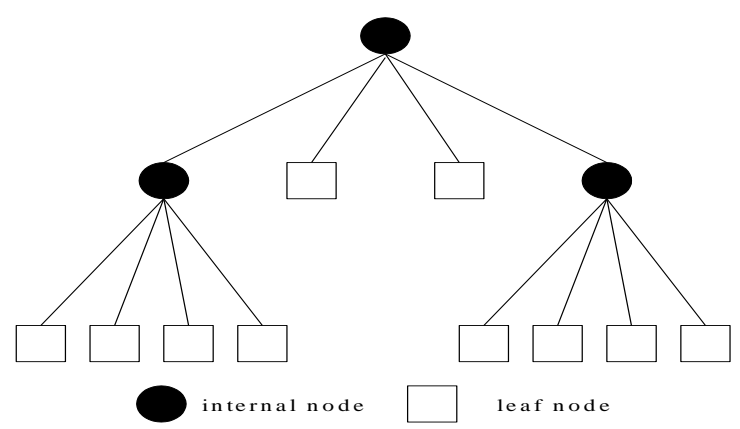

(b) Corresponding three-level quadtree

Figure 3. Example of a Three-level Quadtree

In general, the quadtree segmentation technique is simple and flexible. The question of how many levels should be used is important when performing quadtree segmentation. The more levels a quadtree contains, the more storage cost is required to record the position information of the quadtree structure. 
In the proposed scheme, we use the absolute error between these two AMBTC quantization levels of each image block as the controlling threshold to illustrate the quadtree segmentation process. We assume that the image blocks with sizes larger than $16 \times 16$ are almost heterogeneous in their block activities in most digital images. Therefore, each image to be encoded is segmented into variable-sized blocks by a three-level quadtree.

An example of the quadtree segmentation process is depicted in Figure 3. The original image is listed in Figure 3(a). As shown in Figure 3(b), each internal node in the quadtree has four children emanating from it. In this example, five bits are needed to record the quadtree codes for an image block.

\subsection{The Bit Map Omission Technique}

The bit map omission [18] is a simple technique to reduce bit rates of BTC. The basic idea of the bit map omission technique is to omit the transmission of the bit map if the variance of one given image block is small, or equivalently, if the difference between its two quantization levels $a$ and $b$ is small. Only the block mean should be transmitted as the reconstruction level for the entire block in this case.

Basically, the mean of bit rates achieved by the bit map omission is highly dependent on the given image to be compressed. For most digital images with large uniform areas, this technique achieves a significant bit rate reduction with very little quality loss.

\subsection{Quadtree-segmented AMBTC with Bit Map Omission (AMBTC-QTBO)}

In the proposed scheme, one given color image is first decomposed into three grayscale images. Then, the quadtree segmentation technique is employed to partition each grayscale image into a set of variable-sized blocks. Three-level quadtree structures are imposed on the design of the coding scheme. In other words, only three kinds of blocks sized $16 \times 16,8 \times 8$, and $4 \times 4$ can be found. The reason why only three-level quadtree structures are used instead of four or more levels is that the image blocks with sizes larger than $16 \times 16$ are almost heterogeneous in block activities.

\section{Algorithm of AMBTC-QTBO:}

Step 1: Decompose the color image into three grayscale images.

Step 2: Partition each grayscale image into a set of non-overlapped image blocks of $16 \times 16$ pixels.

Step 3: Compute the block mean and two quantization levels $a, b$ using AMBTC for each $16 \times 16$ image block.

Step 4: If $|a-b| \leq T H Q T$, then encode this $16 \times 16$ block using its block mean and go to Step 8. Otherwise, this block is sub-divided into four equal-size blocks of $8 \times 8$ pixels.

Step 5: Calculate the block mean and two quantization levels $a, b$ using AMBTC for each $8 \times 8$ image block.

Step 6: If $|a-b| \leq T H Q T$, then encode this $8 \times 8$ block using its block mean and go to Step 8. Otherwise, this block is sub-divided into four equal-size blocks of $4 \times 4$ pixels.

Step 7: Calculate these two quantization levels $a, b$ using AMBTC for each $4 \times 4$ block. If $|a-b| \leq T H B O$, then this $4 \times 4$ block is encoded by its block mean. Otherwise, this $4 \times 4$ block is encoded by AMBTC.

Step 8: If there is still any block to be processed, go to Step 3.

In Step 2, each grayscale image is partitioned into a set of non-overlapped image blocks of $16 \times 16$ pixels. The block mean and the quantization levels $a, b$ of each $16 \times 16$ 
image block are calculated. Then, the absolute distance between these two quantization levels is used to determine the block activity. We assume that one given image block is a complex block if a large absolute distance is found. The same statistical measurement is used in the successive quadtree segmentation process for image blocks sized $8 \times 8$ and $4 \times 4$.

In the quadtree segmentation process, the pre-defined threshold $T H Q T$ is used to control whether or not each grayscale image block of $16 \times 16$ or $8 \times 8$ pixels is to be further sub-divided. In addition, another pre-defined threshold $T H B O$ is utilized to determine the block activity for $4 \times 4$ image blocks in the bit map omission technique. After the quadtree segmentation process, the variable-sized blocks will be compressed according to the following rules. For these smooth $16 \times 16$ and $8 \times 8$ blocks, they are encoded by their block means. For these smooth blocks of $4 \times 4$ pixels, the bit plane omission technique is employed to encode them. For the complex blocks of $4 \times 4$ pixels, they are encoded by AMBTC.

Table 1. Storage (unit: bit) Cost for Image Blocks in the Proposed Scheme

\begin{tabular}{|c|c|c|c|c|c|}
\hline \multirow{2}{*}{\multicolumn{2}{|c|}{ Block Size }} & \multirow{2}{*}{$\begin{array}{l}\text { Number of } \\
\text { indicators }\end{array}$} & \multicolumn{2}{|c|}{ Encoded Message } & \multirow{2}{*}{ Total Bits } \\
\hline & & & Block Mean & BTC Trio & \\
\hline \multicolumn{2}{|c|}{$16 \times 16$} & 1 & 8 & N/A & 9 \\
\hline \multicolumn{2}{|c|}{$8 \times 8$} & 2 & 8 & N/A & 10 \\
\hline \multirow{2}{*}{$4 \times 4$} & Smooth & 1 & 8 & N/A & 9 \\
\hline & Complex & 1 & N/A & $(8+8+16)$ & 33 \\
\hline
\end{tabular}

Table 1 lists the storage cost for encoding image blocks of different sizes. To record whether each 16×16 image block is further sub-segmented or not, a 1-bit indicator is needed in the quadtree segmentation process. If one given $16 \times 16$ image block is sub-divided into four $8 \times 8$ image blocks, each of these four equal-size image blocks needs an additional one-bit indicator to record whether it needs to be sub-divided or not. In other words, two bits are needed to record the partition information for each $8 \times 8$ image block. For the $4 \times 4$ image blocks, only a one-bit indicator is required to record the block activity because the total number of $4 \times 4$ blocks can be determined using the partition information of those $16 \times 16$ and $8 \times 8$ blocks.

\section{Experimental Results}

To verify the performance of the proposed scheme, a variety of simulations had been performed on a personal computer with Intel Core i5 $2.8 \mathrm{GHz}$ CPU and $2 \mathrm{~GB}$ random access memory under the operation system of Microsoft Window 7. The experimental results listed below are the average performance of six color $512 \times 512$ images: "Airplane," "House," "Lena," "Pepper," "Splash," and "Tiffany." These testing images are shown in Figure 4. In the simulations, each testing image is partitioned into a set of non-overlapped image blocks of $4 \times 4$ pixels. In other words, there are 16384 image blocks in each testing image.

Results of the image qualities of AMBTC, MPBTC and single bit map AMBTC (AMBTC-SBM) are given in Table 2. Three rules including luminance rule, majority rule, and weighted rule are tested in AMBTC-SBM. The required bit rates of AMBTC and MPBTC are 6 bpp when the block size $4 \times 4$ pixels is used. It is shown that AMBTC achieves better image qualities than MPBTC. The required number of bit rates of AMBTC-SBM equals $4 \mathrm{bpp}$. The image quality loss of more than $3 \mathrm{~dB}$ is incurred by using AMBTC-SBM compared to that by AMBTC. 


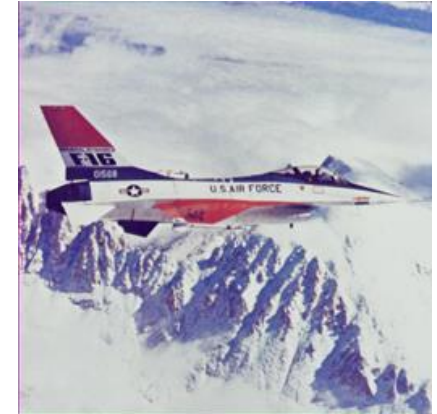

(a) Airplane

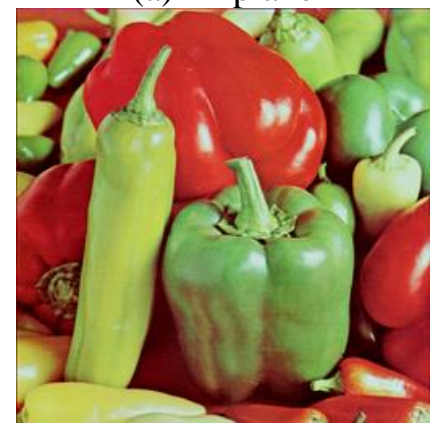

(d) Pepper

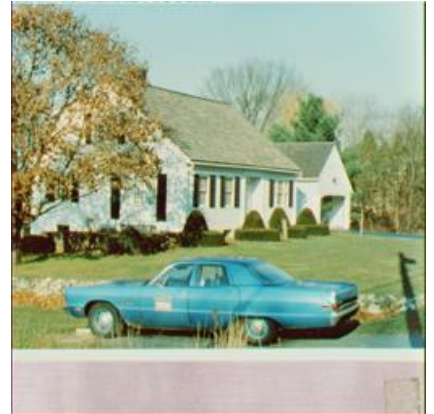

(b) House

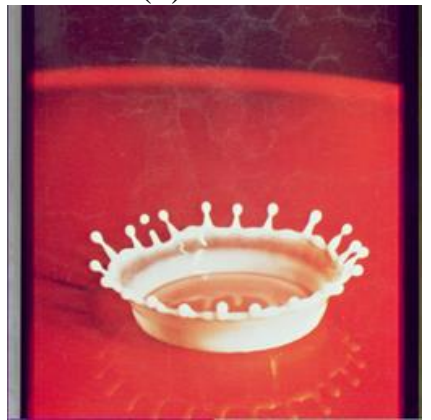

(e) Splash

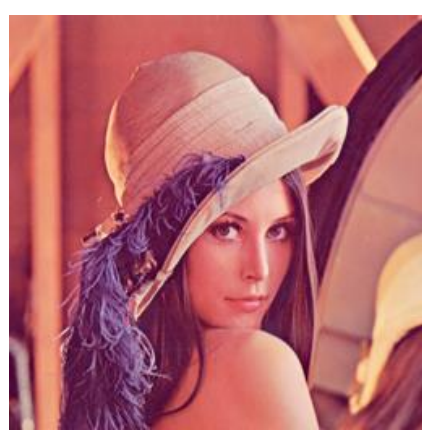

(c) Lena

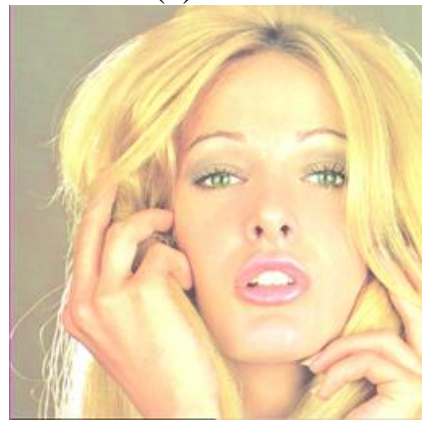

(f) Tiffany

Figure 4. Six Testing Image of $512 \times 512$ Pixels

Table 2. Experimental Results of AMBTC, MPBTC and AMBTC-SBM

\begin{tabular}{|c|c|c|c|c|c|}
\hline \multirow{2}{*}{ Methods } & AMBTC & MPBTC & \multicolumn{3}{|c|}{ AMBTC-SBM (4 bpp) } \\
\cline { 5 - 6 } Images & $(6$ bpp $)$ & $(6$ bpp $)$ & Luminance & Majority & Weighted \\
\hline Airplane & 32.413 & 32.178 & 31.547 & 28.098 & 31.503 \\
\hline House & 30.498 & 30.244 & 28.751 & 28.346 & 28.832 \\
\hline Lena & 33.109 & 32.877 & 31.789 & 31.780 & 31.957 \\
\hline Pepper & 32.701 & 32.464 & 28.981 & 28.174 & 29.547 \\
\hline Splash & 36.158 & 35.946 & 30.422 & 30.338 & 30.741 \\
\hline Tiffany & 35.308 & 35.112 & 29.814 & 27.623 & 29.213 \\
\hline Average & $\mathbf{3 3 . 3 6 5}$ & $\mathbf{3 3 . 1 3 7}$ & $\mathbf{3 0 . 2 1 7}$ & $\mathbf{2 9 . 0 6 0}$ & $\mathbf{3 0 . 2 9 9}$ \\
\hline
\end{tabular}

To understand the performance of the bit map omission technique, experimental results of the AMBTC scheme with the bit map omission technique are given in Figure 5. In the simulations, THBO's are set to $5,10,15, \ldots$, and 30 , respectively. Average results of $33.135 \mathrm{~dB}$ at $4.394 \mathrm{bpp}, 32.072 \mathrm{~dB}$ at $2.754 \mathrm{bpp}$, and $31.229 \mathrm{~dB}$ at $2.335 \mathrm{bpp}$ are obtained by using the AMBTC scheme with the bit map omission technique when THBO's are set to 5,15 , and 25 , respectively.

Experimental results of the image qualities and the bit rates of the proposed scheme without the bit map omission technique are listed in Tables 3 and 4, respectively. In the simulations, THQT's are set to $5,10,15, \ldots$, and 30 , respectively. Average image qualities of $33.331 \mathrm{~dB}, 31.999 \mathrm{~dB}$, and $30.528 \mathrm{~dB}$ are obtained by using the proposed scheme without the bit map omission technique when THQT's are set to 5, 15, and 25, respectively. Average bit rates of $5.451 \mathrm{bpp}, 2.982 \mathrm{bpp}$, and $2.114 \mathrm{bpp}$ are achieved when $T H Q T$ 's are set to 5, 15, and 25, respectively. 


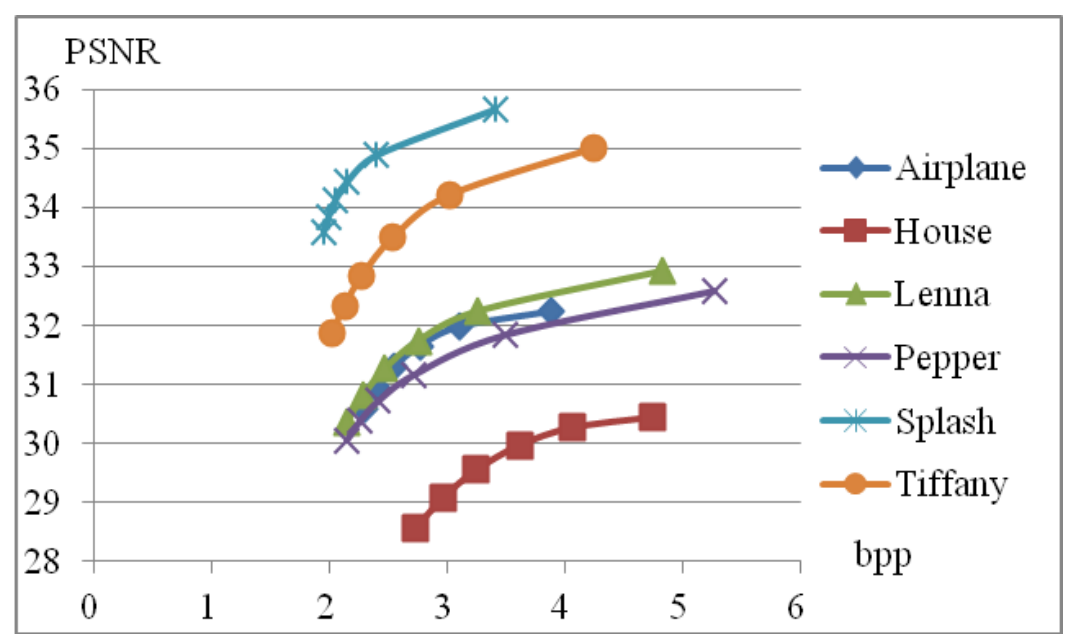

Figure 5. Results of the AMBTC Scheme with the Bit Map Omission Technique

Table 3. Image Qualities of the Proposed Scheme without Bit Map Omission

\begin{tabular}{|c|c|c|c|c|c|c|}
\hline $\begin{array}{c}\text { THQT } \\
\text { Images }\end{array}$ & 5 & 10 & 15 & 20 & 25 & 30 \\
\hline Airplane & 32.279 & 31.868 & 31.539 & 31.054 & 30.498 & 29.938 \\
\hline House & 30.480 & 30.352 & 30.160 & 29.759 & 29.328 & 28.810 \\
\hline Lena & 33.112 & 32.326 & 31.555 & 30.734 & 30.098 & 29.408 \\
\hline Pepper & 32.709 & 32.306 & 31.379 & 30.569 & 29.876 & 29.286 \\
\hline Splash & 36.094 & 34.803 & 33.921 & 33.234 & 32.541 & 32.022 \\
\hline Tiffany & 35.310 & 34.486 & 33.439 & 32.294 & 30.829 & 29.903 \\
\hline Average & $\mathbf{3 3 . 3 3 1}$ & $\mathbf{3 2 . 6 9 0}$ & $\mathbf{3 1 . 9 9 9}$ & $\mathbf{3 1 . 2 7 4}$ & $\mathbf{3 0 . 5 2 8}$ & $\mathbf{2 9 . 8 9 5}$ \\
\hline
\end{tabular}

Table 4. Results of the Bit Rates of the Proposed Scheme without Bit Map Omission

\begin{tabular}{|c|c|c|c|c|c|c|}
\hline $\begin{array}{c}\text { THQT } \\
\text { Images }\end{array}$ & 5 & 10 & 15 & 20 & 25 & 30 \\
\hline Airplane & 4.175 & 2.944 & 2.621 & 2.303 & 2.049 & 1.877 \\
\hline House & 5.103 & 4.353 & 4.003 & 3.59 & 3.278 & 3.000 \\
\hline Lena & 5.989 & 3.669 & 2.786 & 2.247 & 1.947 & 1.695 \\
\hline Pepper & 6.020 & 4.861 & 3.475 & 2.752 & 2.316 & 2.056 \\
\hline Splash & 5.555 & 2.793 & 2.037 & 1.66 & 1.423 & 1.272 \\
\hline Tiffany & 5.865 & 3.929 & 2.970 & 2.277 & 1.668 & 1.303 \\
\hline Average & $\mathbf{5 . 4 5 1}$ & $\mathbf{3 . 7 5 8}$ & $\mathbf{2 . 9 8 2}$ & $\mathbf{2 . 4 7 2}$ & $\mathbf{2 . 1 1 4}$ & $\mathbf{1 . 8 6 7}$ \\
\hline
\end{tabular}

Experimental results of the proposed scheme are given in Figure 6. In the simulations, $T H Q T$ 's are set to $5,10,15, \ldots$, and 30 , respectively. In addition, $T H B O$ 's are set to 5,10 , $15, \ldots$, and 30 , respectively. According to the results, the reconstructed image qualities decrease as THQT increases in the proposed scheme. Similarly, the reconstructed image qualities decrease as $T H B O$ increases in the proposed scheme. However, the required bit rates decrease as THQT increases and as THBO increases. Average results of $33.171 \mathrm{~dB}$ at $4.407 \mathrm{bpp}, 32.332 \mathrm{~dB}$ at $2.683 \mathrm{bpp}, 31.514 \mathrm{~dB}$ at $1.980 \mathrm{bpp}, 30.731 \mathrm{~dB}$ at $1.578 \mathrm{bpp}$, $29.950 \mathrm{~dB}$ at $1.309 \mathrm{bpp}$, and $29.266 \mathrm{~dB}$ at $1.118 \mathrm{bpp}$ are obtained by using the proposed scheme. 


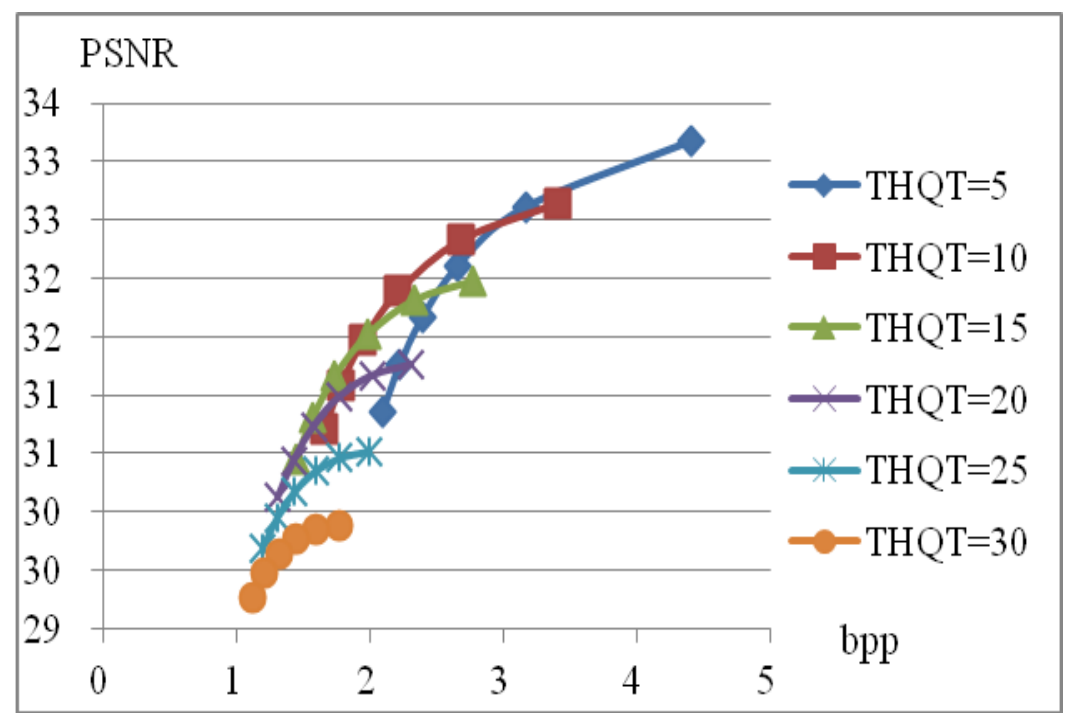

Figure 6. Average Results of the Proposed Scheme

To verify the efficiency of the proposed scheme (AMBTC-QTBO), the rate-distortion curves of the comparative methods are listed in Fig. 7. Among these comparative methods, CICMPBTC [30] and TLAMBTC [31] are based on a single bit map BTC. AMBTC-BPC is an improved coding scheme based on AMBTC. According to the results, it is obvious that the proposed scheme achieves better performance than both CICMPBTC and TLAMBTC. AMBTC-QTBO provides approximately the same performance as AMBTC-BPC. However, AMBTC-QTBO consumes less computational cost than AMBTC-BPC. That is because the block prediction coding process needs to find out whether a similar encoded block exists or not. In other words, the quadtree segmentation process used in the proposed scheme is much simpler than the block prediction process in AMBTC-BPC. According to the results, the state-of-art coding scheme JPEG achieves the best performance of these comparative methods.

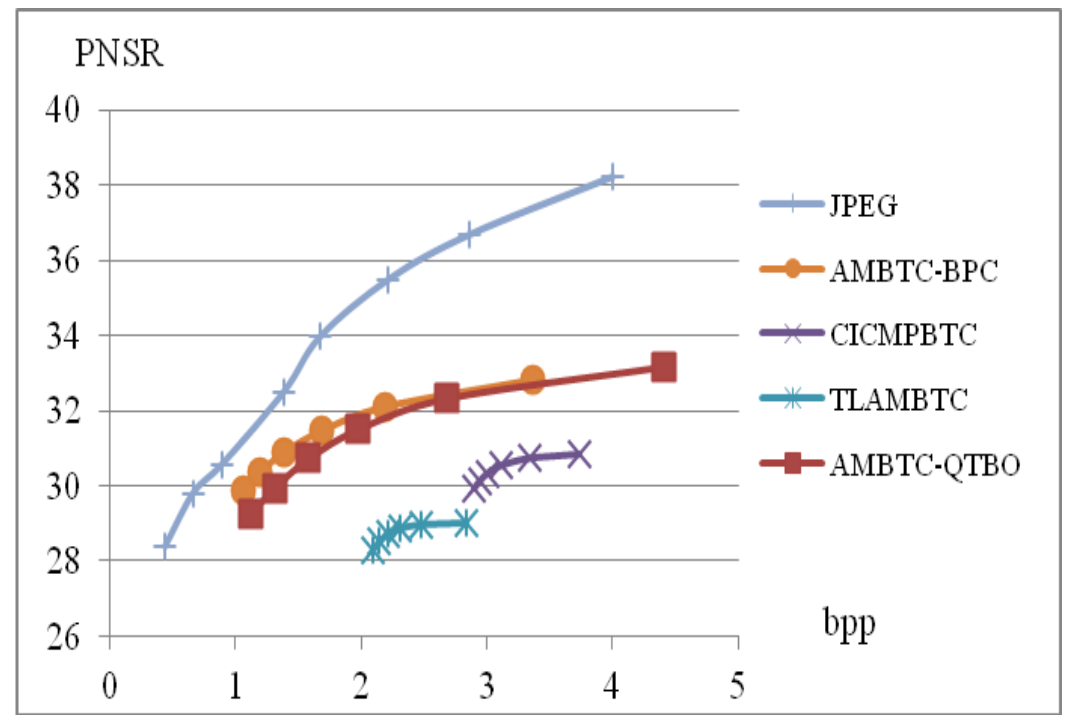

Figure 7. Rate-distortion Curves of the Proposed Scheme and other Comparative Methods 


\section{Conclusions}

In this paper, a novel color image compression scheme based on BTC had been proposed. In the proposed scheme, AMBTC instead of MPBTC is employed because AMBTC is proven to be the optimal scheme based on squared Euclidean distance measurement. In addition, the quadtree segmentation technique and the bit map omission technique are used in the proposed scheme to cut down the bit rates.

The selection of control threshold values for THQT and THBO has great influences on the performance of the proposed scheme. Basically, the threshold values of THQT and $T H B O$ should be set according to the user's requirements. To achieve a better image quality using the proposed scheme, small values of THQT and THBO should be used at the cost of higher bit rates. By choosing adequately controlling thresholds, good image qualities of the compressed images can be obtained by the proposed scheme while keeping low bit rates.

\section{Acknowledgements}

This research was supported by National Science Council, Taipei, R.O.C. under contracpt NSC 101-2221-E-126-014 and NSC 102-2221-E-126-010.

\section{References}

[1] C. C. Chang and Y. C. Hu, "A fast codebook training algorithm for vector quantization", IEEE Transactions on Consumer Electronics, vol. 44, no. 4, (1998), pp. 1201-1208.

[2] Y. C. Hu and C. C. Chang, "Low complexity index-compressed vector quantization for image compression”, IEEE Transactions on Consumer Electronics, vol. 45, no. 1, (1999), pp. 1225-1233.

[3] Y. C. Hu and C. C. Chang, "Variable rate vector quantization scheme based on quadtree segmentation", IEEE Transactions on Consumer Electronics, vol. 45, no. 2, (1999), pp. 310-317.

[4] Y. C. Hu and C. C. Chang, "An effective codebook search algorithm for vector quantization", Imaging Science Journal, vol. 51, no. 4, (2003), pp. 221-234.

[5] Y. C. Hu, B. H. Su and C. C. Tsou, "Fast VQ codebook search algorithm for grayscale image coding", Image and Vision Computing, vol. 26, no. 5, (2008), pp. 657-666.

[6] Y. C. Hu, W. L. Chen, C. C. Lo and J. C. Chung, "An improved vector quantization scheme for grayscale image compression”, Opto-Electronics Review, vol. 20, no. 2, (2012), pp. 187-193.

[7] Y. C. Hu, C. H. Wen, C. C. Lo and W. L. Chen, "Image vector quantization using geometric transform and lossless index coding", Optical Engineering, article no. 037402, vol. 52, no. 3, (2013)

[8] Y. C. Hu, W. L. Chen, C. C. Lo, C. M. Wu and C. H. Wen, "Efficient VQ-based image coding scheme using inverse function and lossless index coding", Signal Processing, vol. 93, no. 9, (2013), pp. 2432-2439.

[9] Y. C. Hu and B. H. Su, "Accelerated k-means clustering algorithm for color image quantization", Imaging Science Journal, vol. 56, no. 1, (2008), pp. 29-40.

[10] Y. C. Hu and B. H. Su, "Accelerated pixel mapping scheme for color image quantization", Imaging Science Journal, vol. 56, no. 2, (2008), pp. 67-78.

[11] Y. C. Hu, M. G. Lee and P. Y. Tsai, "Color palette generation schemes for color image quantization", Imaging Science Journal, vol. 57, no. 1, (2009), pp. 46-59.

[12] Y. C. Hu, C. Y. Li, J. C. Chuang and C. C. Lo, "Variable-rate color image quantization based on quadtree segmentation", Opto-Electronics Review, vol. 19, no. 3, (2011), pp. 282-289.

[13] Y. C. Hu, C. Y. Chiang, W. L. Chen and W. K. Chou, "Lossless index coding for indexed color images", Imaging Science Journal, vol. 60, no. 1, (2012), pp. 54-63.

[14] Y. C. Hu, W. L. Chen, C. C. Lo and C. M. Wu, "On a predictive scheme for color image quantization", Opto-Electronics Review, vol. 20, no. 2, (2012), pp. 159-167.

[15] Y. C. Hu, W. L. Chen, B. H. Su and W. K. Chou, "Dynamic sub-range search methods for image color quantization”, Imaging Science Journal, vol. 61, no. 2, (2013) , pp. 80-93.

[16] E. J. Delp and O. R. Mitchell, "Image compression using block truncation coding", IEEE Transactions on Communications, vol. 27, no. 9, (1979), pp. 1335-1342.

[17] P. Franti, P. Nevalainen abd T. Kaudoranta, "Compression of digital image by block truncation coding: a survey”, Computer Journal, vol. 37, no. 4, (1994), pp. 308-332.

[18] M. D. Lema and O. R. Mitchell, "Absolute moment block truncation coding and its application to color image”, IEEE Transactions on Communications, vol. 32, no. 10, (1984), pp. 1148-1157.

[19] D. Halverson, N. Griswold and G. Wise, "A generalized block truncation coding algorithm for image compression", IEEE Transactions on Acoustics Speech Signal Processing, vol. 32, no. 3, (1984), pp. 664-668. 
[20] D. Chen and A. C. Bovik, "Visual pattern image coding", IEEE Transactions on Communications, vol. 38, no.12, (1990), pp. 2137-2146.

[21] P. Nasiopoulos, R. K. Ward and D. J. Morse, "Adaptive compression coding”, IEEE Transactions on Communications, vol. 39, no. 7, (1991), pp. 1245-1254.

[22] B. C. Dhara and B. Chanda, "Block truncation coding using pattern fitting," Pattern Recognition, vol. 37, no. 11, (2004), pp. 2131-2139.

[23] B. Zeng, "Two interpolative BTC image coding schemes", IEE Electronics Letters, vol. 27, no. 13, (1991), pp. 1126-1128.

[24] Y. V. Ramana and C. Eswaran, "A new algorithm for BTC bit plane coding”, IEEE Transactions on Communications, vol. 43, no. 6, (1995), pp. 2010-2011.

[25] J. Vaisey and A. Gersho, "Image compression with variable block size segmentation", IEEE Transactions on Signal Processing, vol. 40, no. 8, (1992), pp. 2040-2060.

[26] Y. C. Hu, "Low-complexity and low-bit-rate image compression scheme based on AMBTC", Optical Engineering, vol. 42, no. 7, (2003), pp. 1964-1975.

[27] Y. C. Hu, "Improved block truncation coding for image compression", IEE Electronics Letters, vol. 39, no.19, (2003), pp. 1377-1379.

[28] Y. C. Hu, "Predictive moment preserving block truncation coding for gray-level image compression", Journal of Electronic Imaging, vol. 13, no. 4, (2004), pp. 871-874.

[29] Y. Wu and D. C. Coll, "Single bit-map block truncation coding of color images", IEEE Journal on Selected Areas in Communications, vol. 10, no. 5, (1992), pp. 952-959.

[30] C. K. Yang, J. C. Lin and W. H. Tsai, "Color image compression by moment-preserving and block truncation coding techniques", IEEE Transactions on Communications, vol. 45, no.12, (1997), pp. 1513-1516.

[31] C. C. Chang, T. S. Chen and J. C. Chung, "A colour image compression scheme based on two layer absolute moment block truncation coding", Imaging Science Journal, vol. 48, no. 2, (2000), pp. 53-62.

[32] Y. C. Hu, B. H. Su and P. Y. Tsai, "Coluor image coding scheme using absolute moment block truncation coding and block prediction technique”, Imaging Science Journal, vol. 56, no. 5, (2008), pp. 254-270.

\section{Authors}

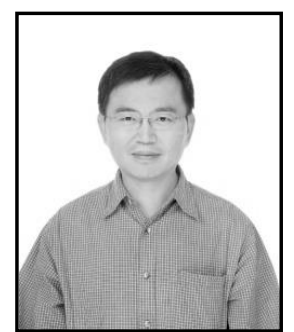

Wu-Lin Chen is currently an associate professor in the department of Computer Science and Information Management at Providence University. He received his M.S. and Ph.D. degrees in the School of Industrial Engineering at Purdue University in 1995, and 1999, respectively. His research interests include operations research, production management, stochastic models, and image processing.

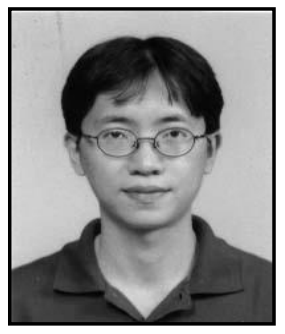

Yu-Chen Hu is a professor in the Department of Computer Science and Information Management, Providence University, Sha-Lu, Taiwan. He is a member of ACM and IEEE. Dr. Hu Servers as the Editor-in-Chief of International Journal of Image Processing since 2009. He joints the editorial boards of several other journals including International journal of Security and Its Applications, International Journal of Signal Processing, Image Processing and Pattern Recognition, International Journal of Digital Contents and Applications and so on. His research interests include image and signal processing, data compression, information hiding, and data engineering.

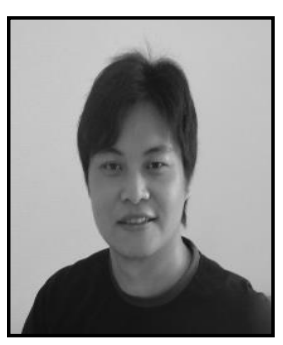

Kuo-Yu Liu is an assistant professor in the Department of Computer Science \& Communication Engineering at Providence University, Taiwan. He is the deputy director of the CALL research center. His research interests include e-learning technologies and the integration of educational model, game-based learning and cross-media analysis. 


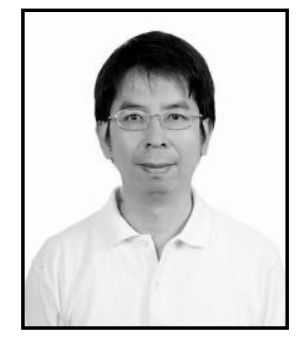

Chun-Chi Lo received the B.S., M.S., and Ph.D. degrees in computer science and information engineering from the National Taiwan University, Taipei, Taiwan, R.O.C., in 1989, 1991, and 1996, respectively. In 2004, he joined the faculty of the Department of Computer Science and Information Engineering, Providence University, Taiwan. Currently, he is an Assistant Professor. His research interests include wireless sensor networks, mean-field annealing, combinatorial optimizations, and image processing.

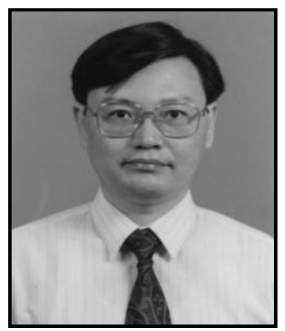

Chia-Hsien Wen is currently an associate professor of the Department of Computer Science and Information Management, Providence University, Taiwan. Prior to joining Providence University in 2005, he was the director of the Computing Center at Taichung Veterans General Hospital (TCVGH). His research interests include medical image processing, medical informatics, database management, and machine learning. 